\title{
Evidence for Free Rotation Restriction of Unsaturated Bond in Aggregation-Induced Emission
}

\author{
Song Zhang ${ }^{1,}$, Lian Wang ${ }^{1,2}$, Miaomiao Zhou ${ }^{1,2}$, and Bing Zhang ${ }^{1}$ \\ ${ }^{1}$ State Key Laboratory of Magnetic Resonance and Atomic and Molecular Physics, Wuhan Institute of \\ Physics and Mathematics, Chinese Academy of Sciences, Wuhan 430071, China \\ ${ }^{2}$ University of Chinese Academy of Sciences, Beijing 100049, China
}

\begin{abstract}
We experimentally demonstrate that that nonradiative process, which competes with radiative decay, involves two main stages, namely the restricted intramolecular rotation and internal conversion process. The free rotation restriction of the unsaturated bond at the excited state is the key factor for AIE effects.
\end{abstract}

\section{Introduction}

Aggregation-induced emission (AIE) phenomena have been attracting more and more interest due to the colossal potential in chemo/biosensor, solid emitter, bioimaging, multistimuli response systems, and so on [1-3]. However, due to the diversity and complexity of AIEgen systems, the mechanism that leads to AIE is also sophisticated and uncertain from time to time for some specific AIE molecules. Up to now, no any direct and convictive evidence has been provided to prove the rotation mechanism of the unsaturated bond at the excited state for AIEgen system. We can discuss fluorescence or fluorescence enhancement not only from RIR by intermolecular aggregation effect but also from RIR by intramolecular rotation fixation. While the intramolecular rotation is generally related to the rotation of a single bond, this work also speculated that the rotation of the unsaturated bond at the excited state would result in the fluorescence quenching of the AIEgen system in solution.

\section{Experiment}

Ultrafast broadband absorption measurements were performed based on a Ti:sapphire femtosecond laser system [4]. Briefly, the seed beam is generated by a commercial Ti:sapphire oscillator pumped by a CW second harmonic of an Nd:YVO4 laser, and then amplified by an $\mathrm{Nd}$ :YLF pumped regenerative amplifier to generate a $1 \mathrm{kHz}$ pulse train centered at $800 \mathrm{~nm}$ of approximately $35 \mathrm{fs}$ pulse width and with maximum energy of 1 $\mathrm{mJ} /$ pulse. The $400 \mathrm{~nm}$ and $350 \mathrm{~nm}$ were used as pump pulses. $400 \mathrm{~nm}$ is the second

\footnotetext{
${ }^{*}$ Corresponding author: zhangsong@,wipm.ac.cn
} 
harmonic generation of the fundamental pulse obtained by a $0.5 \mathrm{~mm}$ thick BBO crystal, with pulse energy $\sim 2 \mu \mathrm{J}$ at the sample position. Pump pulse at $350 \mathrm{~nm}$ was produced from a homemade NOPA system and attenuated to $\sim 2 \mu \mathrm{J}$ in the experiments. The NOPA pulse needs to be temporally compressed in order to obtain the minimum pulse width compatible with their bandwidth. A white light continuum generated by focusing the fundamental light on a $1 \mathrm{~mm}$ sapphire plate was reflected from the front and back surfaces of a quartz plate to obtain the probe and reference beams. The pump and probe pulses intersect in the sample at an angle of $\sim 4^{\circ}$, and the reference beam is transmitted through the sample at a different spot. The relative polarization of the pump and probe pulses is set to the magic angle for all the measurements. A linear translation stage is used to delay the probe beam to monitor the pump-probe dynamics. The resulting spectra are detected by a CCD camera equipped with a spectrometer. The instrumental response function of the system was determined by cross correlation between the excitation and probe pulses using the optical Kerr-gate method. All quantum chemical calculations are performed using the Gaussian $09 \mathrm{~W}$ suit of program. The geometries of the ground and excited states of AIEgen system were optimized using the DFT and TDDFT with B3LYP functional and $6-31+\mathrm{G}(\mathrm{d}, \mathrm{p})$ basis set in gas phase and methanol, DMSO, and aniline solutions, respectively.

\section{Results and Discussion}

The transient absorption spectra of HPS in methanol, DMSO, and aniline were obtained from 450 to $700 \mathrm{~nm}$, respectively. The viscosities of three solvents used in this work are distinguishable, while the polarities are similar. In all the time-resolved spectra, there exist obviously two main broad bands of a positive signal. Taking the spectrum in DMSO as an example, it is noticed that the band centred at $\sim 490 \mathrm{~nm}$ quickly increased to the maximum in the first 20 ps and then slowly decayed, as shown in Fig. 1. The dynamic decay process was taken into account with two typical time components $\tau_{1}$ and $\tau_{2}$, which were measured to be 16 and $76 \mathrm{ps,} \mathrm{respectively.} \mathrm{The} \mathrm{short} \mathrm{lifetime} \mathrm{component} \mathrm{can} \mathrm{be}$ attributed to the geometry relaxation from Franck-Condon configuration to the intrarotational structure. The rising components in methanol and aniline were measured to be 6.5 and $48 \mathrm{ps}$. On the whole, it is obviously that the lifetime becomes longer from several to tens of picosecond with the increase of the solvent viscosity. The decay component $\tau_{2}$ is measured to be 19, 76 and 87 ps in methanol, DMSO, and aniline, respectively.
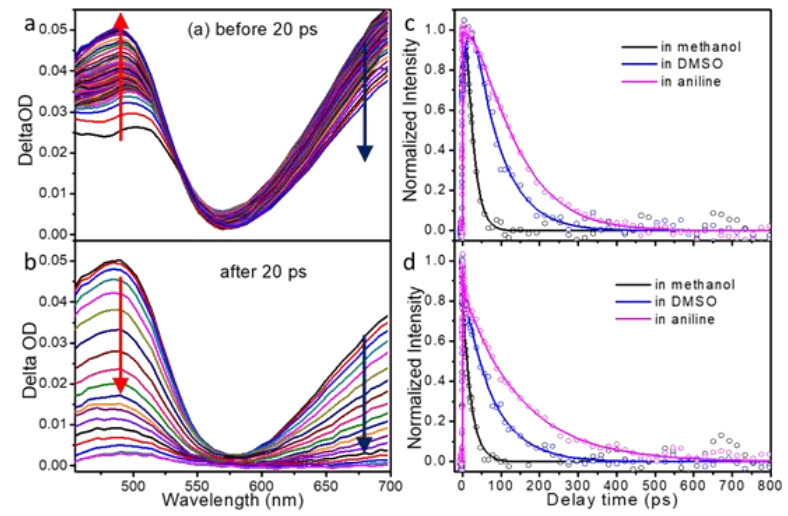

Fig. 1. Time resolved spectra of HPS in DMSO. (a) before 20 ps; (b) after 20 ps. The experimental (dotted lines) and fitted (solid lines) data are normalized decay curves of the two absorption bands at (c) $480 \mathrm{~nm}$ and (d) $665 \mathrm{~nm}$, respectively. 
Furthermore, femtosecond transient absorption spectra of cis-TPE dicycles and gem-one were also measured following $350 \mathrm{~nm}$ excitation [5]. The dynamic decay process was also taken into account with two typical time components. The band at long wavelength was ascribed to the absorption of the planar excited states of cis $^{*}$ or gem ${ }^{*}$ and the other at short wavelength belonged to the twisted excited state cis $^{* *}$ or gem $^{* *}$, which resulted from rotation of the unsaturated bond at the excited state for about $90^{\circ}$. In time-resolved spectra, the species at short wavelength had an obviously growing process and the increase ended up in less than $20 \mathrm{ps}$, hinting that the twisted excited state originated from the planar excited state. Therefore, both the TPE dicycles displayed unsaturated bond rotation at the excited state. In addition, the component $\tau_{3}$ of gem-isomer, which indicated the rotation, was measured to be 6 ps and obviously faster than 15 ps of cis-one. It could be envisioned that the rotation in the gem-isomers was freer and could be implemented from $0-360^{\circ}$, while it was difficult to excess $90^{\circ}$ in cis-isomers due to the bridge restriction. The absorption spectra of cis-isomer and gem-isomer at the excited state reached their maximum intensity at 21 and $14 \mathrm{ps,}$, respectively, indicating the accomplishment of the rotation. Moreover, the absorption maximum wavelength of the gem-isomer is blue-shifted about $15 \mathrm{~nm}$ relative to that of cis-isomer. From the absorption spectra, meanwhile, the area ratio of redband of gem-isomer to the blue one was much less than the ratio of cis-isomer. This result further corroborated that the unsaturated bond of gem-isomer rotated more freely than that of cis-isomer. Therefore, with more free rotation at the excited state, gemisomer relaxed more through radiationless processes and showed lower fluorescence quantum yield than cis-one.

\section{Conclusion}

In conclusion, TPE dicycles, especially cis- and gem-isomer which were isomers with completely the same elements and substituents, demonstrated by their photophysical properties that the rotation inhibition of the double bond at the excited state also plays a key role in the AIE effect. This provides, for the first time, persuasive examples of the AIE effect from free rotation restriction of the unsaturated bond at the excited state.

We acknowledge financial support by the National Natural Science Foundation of China (Grant Nos. 11674355, 21573279).

\section{References}

1. J. Luo, Z. Xie, J. W. Y. Lam, L. Cheng, H. Chen, C. Qiu, H. S. Kwok, X. Zhan, Y. Liu, D. Zhu, B. Z. Tang, Chem. Comun. 1740 (2001).

2. X. Lim, Nature. 531, 26 (2016).

3. R. Hu, N. L. C. Leung, B. Z Tang, Chem. Soc. Rev. 43, 4494 (2014).

4. S. Zhang, S. Sun, M. Zhou, L. Wang and B. Zhang, Sci. Rep. 7, 43419 (2017).

5. J. Xiong, Y. Yuan, L. Wang, J. Sun, W. Qiao, H. Zhang, M. Duan, H. Han, S. Zhang, Y. Zheng, Org. Lett. 20, 373 (2018). 